\section{Tratamiento estético en fluorosis dental grado 3 según índice Thylstrup y Fejerskov, mediante métodos conservadores}

\section{Aesthetic treatment in grade three dental fluorosis of Thylstrup-Fejerskov index, using conservative methods}

\begin{abstract}
Resumen
La ausencia de armonía en la sonrisa de un paciente constituye una causa por la cual su autoestima y comportamiento con su entorno se ven afectados. Ecuador ha sido considerado una zona endémica de fluorosis, desencadenando alteraciones dentales estéticas y funcionales; la fluorosis se presenta como manchas opacas que siguen un patrón horizontal en su fase temprana y manchas marrones y pérdida de estructura en estado avanzado. El presente caso tiene como objetivo exponer el caso de un paciente joven, diagnosticado con fluorosis grado 3 según el índice Thylstup y Fejerskov (TF), en el cual se realizó blanqueamiento con peróxido de hidrógeno al $40 \%$, seguido de blanqueamiento casero con peróxido de carbamida al 35\%, 20 minutos diarios por 7 días. La combinación de procedimientos de blanqueamiento resultó idónea en pacientes con fluorosis grado 3 según el índice de TF, mimetizando el moteado característico de la fluorosis dental mejorando así la estética del paciente y garantizando el éxito del presente caso. La aplicación de tratamientos conservadores, es eficaz, para restablecer estética dental, función y confianza en el paciente al momento de sonreír.
\end{abstract}

Palabras clave: Blanqueamiento dental; Estética dental; Fluorosis dental; Tratamiento conservador (fuente: DeCS BIREME).

\begin{abstract}
The lack of harmony in a patient's smile affects their self-esteem and behavior with their environment. Ecuador has been considered an endemic zone of fluorosis, triggering aesthetic and functional dental alterations. Fluorosis appears as opaque spots that follow a horizontal pattern in its early phase and brown spots and loss of structure in an advanced stage. In this article we present the case of a young patient, diagnosed with dental fluorosis grade three of Thylstup-Fejerskov (TF) index, treated with $40 \%$ hydrogen peroxide bleaching, followed by $35 \%$ peroxide carbamide home whitening for 30 minutes daily, during 7 days. The combination of whitening procedures was ideal to mimic the mottling characteristic of dental fluorosis, thus improving the aesthetics of the patient and guaranteeing the success of the present case. The use of a conservative
\end{abstract}

\section{Caso Clínico}

Jerely Normanda Chico Lara ${ }^{1, a}$, Johanna Sabrina Coello González ${ }^{2, a}$, Víctor Alfonso Montaño Tatés ${ }^{1, a}$, Jorge Eduardo Carrillo Sandoval 1,a, Ana del Carmen Armas Vega ${ }^{1, b}$

${ }^{1}$ Universidad UTE, Quito, Ecuador.

2 Universidad Central del Ecuador, Quito, Ecuador.

a Odontólogo general.

${ }^{\mathrm{b}} \mathrm{PhD}$ en dentística restauradora.

\section{Correspondencia:}

Chico Lara Jerely: jery-0895@hotmail.es

Universidad UTE, Quito, Ecuador

ORCID: 0000-0002-6122-0782

\section{Coautores:}

Coello González Johanna: jsjohas91@gmail.com ORCID: 0000-0003-3374-9045

Montaño Tatés Víctor: victor18alfonso@hotmail.com ORCID: 0000-0001-7328-5834

Carrillo Sandoval Jorge: george_0794@hotmail.com ORCID: 0000-0002-0490-1151

Armas Vega Ana: ana del ec@yahoo.es

ORCID: 0000-0003-3800-8166

\section{Editor:}

Yuri Castro-Rodríguez

Universidad Nacional Mayor de San Marcos, Perú.

Conflicto de intereses: los autores declaran no poseer conflictos de interés relacionado con este caso clínico.

Fuente de financiamiento: autofinanciada

\section{Recibido: 24/01/20}

Aceptado: $18 / 05 / 20$

Publicado: 04/08/20 
treatment was effective to restore dental aesthetics, function and confidence of the patient when smiling.

Keywords: Teeth whitening; Dental esthetics; Dental fluorosis; Conservative treatment (source: MeSH NLM).

\section{Introducción}

La fluorosis dental (FD), constituye un defecto del desarrollo del esmalte dental causado por la ingesta crónica y excesiva de fluoruros, que desencadenan un esmalte hipomineralizado y poroso ${ }^{1}$, manifestándose clínicamente con manchas blancas y opacas que siguen un patrón lineal horizontal; en estado avanzado se presenta como manchas marrones, aumento de la porosidad y afectación parcial o total la superficie del esmalte ${ }^{2}$.

La elevada presencia de FD tiene repercusión estética y psicológica en quienes la presentan ${ }^{3}$. Así, el objetivo del presente caso es exponer la solución estética mínimamente invasiva, en un paciente con problemas estéticos debido a la presencia de fluorosis. Tanto la belleza como la naturalidad al sonreír son juzgadas por las personas; estudios demuestran que una persona con sonrisa agradable tiene un mejor desempeño social y por ende una mejor calidad de vida ${ }^{4}$; es por esto que los pacientes buscan tratamientos estéticos basados en un estereotipo social ${ }^{5}$.

Existen diversos criterios para evaluar el grado de severidad de FD, de los cuales, el índice de Dean, y el índice de Thylstup y Fejerskov (TF), son los más relevantes al momento de realizar su diagnóstico; el primero descrito en 1934, el cual evaluando dos de los dientes más afectados, clasificó la patología en cinco tipos diferentes ${ }^{6}$ (Tabla 1). Esta clasificación sigue siendo el "estándar de oro", aunque se han desarrollado otros índices, como el de TF considerado completo, por evaluar la FD en todos los niveles de severidad, se basa en los diferentes grados histopatológicos propios de la $\mathrm{FD}^{7}$ y en los cambios adamantinos que se observan en la superficie dental en diez diferentes categorías lo que permite la determinación de niveles leves a niveles más severos ${ }^{8}$ (Tabla 2).

En la actualidad existe mucha evidencia científica de cómo resolver problemas estéticos en cuanto FD, el tratamiento siempre dependerá del diagnóstico '; los grados de fluorosis leves se podrán tratar con procedimientos mínimamente invasivos y en casos de fluorosis severas su tratamiento será medianamente invasivo ${ }^{10,11}$. En casos leves se puede optar por blanqueamiento o una microabrasión ${ }^{12}$. En casos moderados se puede realizar microabrasión combinada con blanqueamiento, macroabrasión de forma selectiva, restauraciones de

Tabla 1. Índice de Dean

Normal.- La superficie del esmalte es suave, brillante y habitualmente de color blanco-cremoso pálido.

Muy leve.- Pequeñas zonas blancas como papel y opacas dispersas irregularmente en el diente, pero que afectan a menos de $25 \%$ de la superficie dental labial.

Leve.- La opacidad blanca del esmalte es mayor que la correspondiente a la muy ligera, pero abarca menos de $50 \%$ de la superficie dental labial.

Moderada.- La superficie del esmalte de los dientes muestra un desgaste marcado, además el tinte pardo es con frecuencia una característica que la distingue.

Severa.- La superficie del esmalte está muy afectada y la hipoplasia es tan marcada que puede afectarse la forma general del diente. Se presentan zonas excavadas o gastadas y se halla un extendido tinte pardo; los dientes a menudo presentan un aspecto corroído.

Tabla 2. Índice de Thylstrup and Fejerskov

TF0.- Se caracteriza por esmalte normal, liso, traslúcido y cristalino de color uniforme. Estas características permanecen aún después del secado con aire prolongado.

TF1.- Esmalte liso, traslúcido y cristalino, con finas bandas horizontales de color blanquecino.

TF2.- Esmalte liso, traslúcido y cristalino acompañado con gruesas líneas horizontales blanquecino.

TF3.-Esmalte liso, translúcido y cristalino. Acompañado por gruesas líneas opacas blanquecinas, con manchas opacos que pueden ir del color amarillo al café.

TF4.- Toda la superficie tiene una marcada opacidad que varía del blanco opaco al gris. Pudiendo estar acompañada de vetas de color amarillo o café. También pueden aparecer partes del esmalte desgatadas por atrición.

TF5.- Superficie totalmente opaca, con pérdida del esmalte en forma de cráter no mayor a $2 \mathrm{~mm}$ de diámetro. Las pigmentaciones suelen asentarse en el fondo del cráter y por lo general son extrínsecas.

TF6.- Superficie blanca opaca con mayor cantidad de cráteres, que al unirse va formando bandas horizontales de esmalte faltante. Las pigmentaciones suelen asentarse en el fondo del cráter y por lo general son extrínsecas.

TF7.- Superficie totalmente blanca opaca con pérdida de superficie de esmalte en áreas irregulares, iniciando en el tercio incisal/oclusal, menor al $50 \%$ de la superficie del esmalte.

TF8.- Pérdida de la superficie del esmalte que abarca más de un 50\%. El remanente del esmalte es blanco opaco. Suele haber exposición de dentina con lesiones de caries.

TF9.- Pérdida de la mayor parte de la superficie de esmalte. Dentina expuesta. 
resina compuesta y en la actualidad la aplicación de resinas infiltrantes ${ }^{12,13}$, y en casos severos lo más común será emplear restauraciones indirectas de recubrimiento parcial o total, tomando en cuenta el grado de pérdida de la estructura dental y en ciertos casos restauraciones directas mediante resina compuesta ${ }^{14}$.

El blanqueamiento dental es uno de los procedimientos estéticos más solicitados por los pacientes ${ }^{15}$ pero se debe valorar cada caso ya que habrán pacientes donde el blanqueamiento no es el procedimiento más indicado; existen criterios para la no aplicación de un tratamiento aclarante, dentro de las cuales son, las caries activas ${ }^{16}$, presencia de enfermedad periodontal, exposición dentinaria, microfracturas del esmalte por donde podría penetrar el agente aclarador y causar daño pulpar, entre otros ${ }^{17}$ Se deben considerar también los hábitos del paciente, por ejemplo en pacientes fumadores el blanqueamiento dental no está contraindicado, pero no tendrá una estabilidad adecuada a largo plazo ${ }^{18}$.

Existen tres técnicas para realizar blanqueamiento dental, el blanqueamiento de consultorio, el de uso domiciliario y una combinación de los dos; los agentes aclaradores comúnmente usados son el peróxido de hidrógeno y el peróxido de carbamida ${ }^{19}$. El blanqueamiento de consultorio emplea concentraciones elevadas de peróxido de hidrógeno por lo cual acelera el proceso, donde una sesión de 30 - 40 minutos de acuerdo a las instrucciones del fabricante será más que suficiente. El blanqueamiento de aplicación domiciliaria emplea concentraciones muy bajas de peróxido de hidrógeno y peróxido de carbamida, siendo el segundo agente uno de los más seguros, la literatura recomienda que se puede enviar por un máximo de 3 semanas de forma segura, realizando controles cada 8 días en el consultorio ${ }^{20}$.

Finalmente, la técnica combinada, como su nombre lo indica, se realiza una aplicación en consultorio y 15 días de aplicación en el domicilio el agente de baja concentración, con un máximo de 45 minutos diarios que es lo que dura el efecto de estos agentes, reduciendo así la sensibilidad dental y mejorando el efecto estético ${ }^{21}$.

En algunos casos puede producirse hipersensibilidad durante o después del tratamiento, esta puede ser muy leve en algunos pacientes o tener un efecto exacerbado en otros, pero normalmente es un efecto tratable y reversible ${ }^{22}$. La hipersensibilidad puede tratarse con la aplicación local de geles fluorados o nitrato de potasio, siendo estos agentes desensibilizantes que taponan los túbulos dentinarios expuestos o bloquean el impulso nervioso respectivamente. Es importante las recomendaciones que se envían al paciente como el evitar consumir ciertos tipos de comidas o bebidas ${ }^{23,24}$.

\section{Reporte del caso}

Paciente masculino de 24 años de edad, acude a consulta odontológica de la Universidad UTE, sede Quito - Pichincha, con motivo de consulta: "No me siento satisfecho con el color de mis dientes, quisiera tenerlos uniformes y blancos", expresando inconformidad estética dental debido a la presencia de manchas blancas opacas, con áreas amarillentas compatibles con fluorosis dental, manifestando su necesidad de conseguir un color blanco y uniforme. Paciente afirma residir desde su nacimiento en Cotopaxi provincia de la sierra central del Ecuador, considerada endémica de FD ${ }^{25}$. Paciente sin antecedentes patológicos sistémicos importantes, al realizar el diagnóstico clínico no se evidencia restauraciones, ni caries dental, no presentó enfermedad periodontal, pero si se observó en la gran mayoría de las estructuras dentales un esmalte liso, translúcido, se logra apreciar líneas gruesas opacas de color blanquecino y manchas de color amarillento; el análisis clínico y fotográfico ejecutado permitió diagnosticar al paciente con FD grado 3 según el índice TF (Figura 1).Tras una revisión exhaustiva del

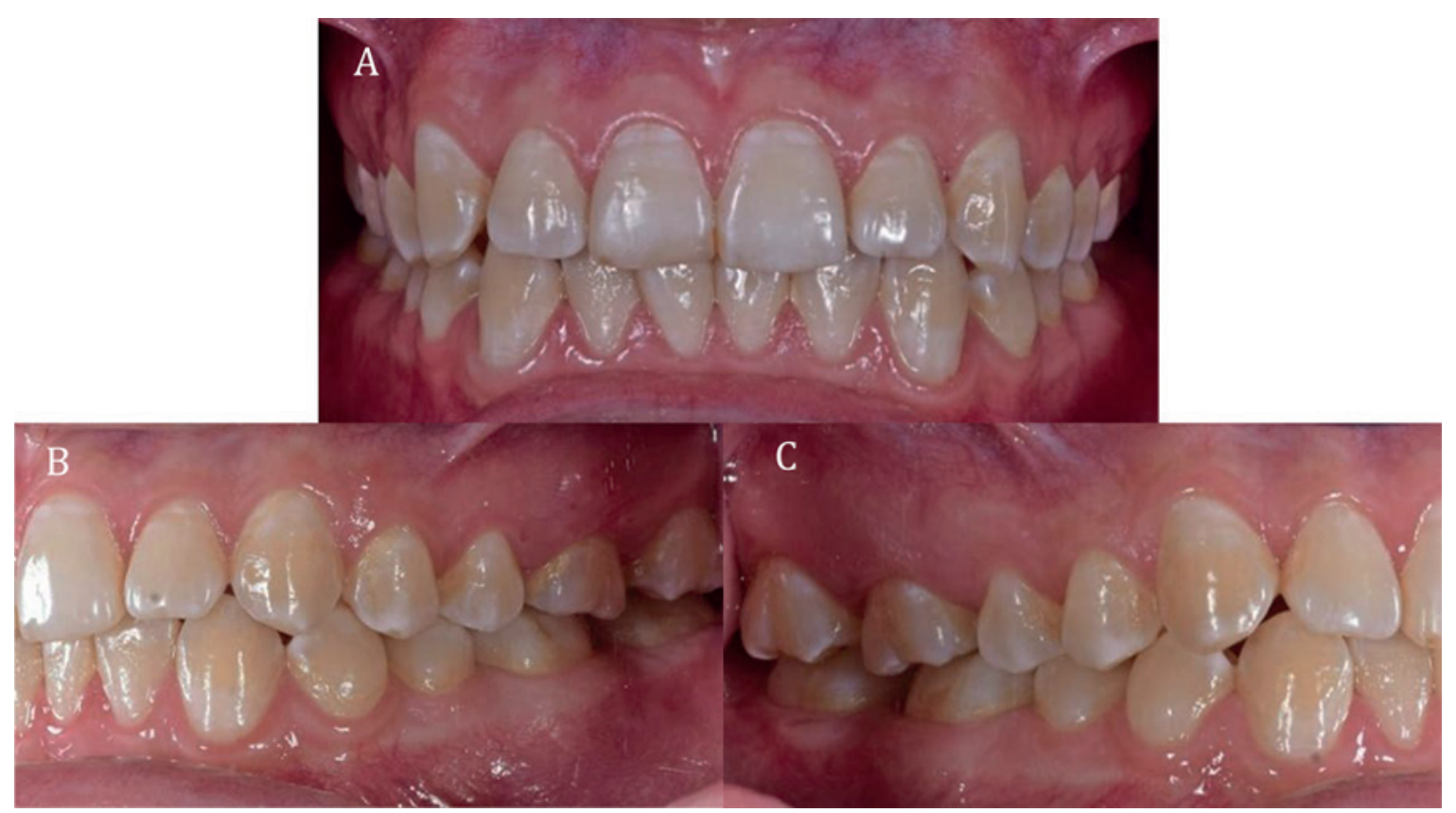

Figura 1. Fotografías intraorales. A. Fotografía frontal. B. Fotografía intraoral izquierda. C. Fotografía intraoral derecha 
caso y de fotografías obtenidas, se explicó al paciente las alternativas de tratamiento junto con los pro y contra de cada procedimiento y su validación mediante consentimiento informado, eligiendo como tratamiento el blanqueamiento clínico y casero.

Previo a la aplicación del blanqueamiento clínico, fue realizada una limpieza de las superficies dentales mediante pasta de piedra pómez, desinfección de la zona a tratar con clorhexidina (Figura 2A). A continuación, se registró el color inicial $2 \mathrm{~A}$, por medio de colorímetro (Chromascop®, Ivoclar Vivadent, USA) y fotografía de registro (Figura 2B). Posteriormente, se realizó la protección de los tejidos blandos con barrera gingival (Top dam ${ }^{\circledR}, F G M$, Brasil) aplicada según las instrucciones del fabricante, se polimerizó el material con una lámpara de fotopolimerización (Led C, Woodpecker ${ }^{\circledR}$, USA) durante 20 segundos (Figura 2C). Se procede a preparar el peróxido de hidrógeno al 40\% (Opalescense Boost $\AA_{\text {, }}$ Ultradent, USA) según indicaciones del fabricante, se aplica el agente blanqueador sobre la superficie vestibular de cada diente en una sola sesión de 20 minutos (Figura 2D). Una vez transcurrido el tiempo de aplicación, se eliminó el producto aclarador mediante succionador con punta intraoral y abundante agua (Figura 2E). Se registró el color obtenido 01 según el colororímetro (Chromascop®, Ivoclar Vivadent, USA), posterior a la aplicación del blanqueamiento clínico (Figura 3).
Concluido el procedimiento de blanqueamiento clínico, y frente al relato por parte del paciente de presencia de sensibilidad, se aplica agente desensibilizante a base de nitrato de potasio (Ultraez ${ }^{\circledR}$, Ultradent, USA) (Figura $4 \mathrm{~A}$ ), siguiendo las recomendaciones del fabricante, posteriormente, se aplicó flúor barniz (Clinpro®, 3M ESPE) con una concentración de 22600 ppm de fluoruro, respetando las indicaciones del fabricante y utilizando la guía de dosificación; se indicó al paciente sobre la dieta e higiene posterior a la colocación del producto. La sensibilidad manifestada por el paciente fue reduciendo casi en su totalidad después de haber aplicado el flúor barniz, una vez aplicado el producto sobre la superficie dental también se promovió la remineralización del esmalte dental, sin perjudicar el tratamiento estético ya que el producto viene en color blanco, casi invisible brindando comodidad al paciente.

Una vez transcurridas 48 horas después del blanqueamiento clínico fue aplicado el blanqueamiento casero a base peróxido de Carbamida al 35\% (Opalescense®, Ultradent, USA) (Figura 4B). Mediante el uso de cubetas personalizadas de acetato semi-rígido, con espacio en vestibular de $0,5 \mathrm{~mm}$ para que se aloje el agente blanqueador, su utilización fue indicada durante 20 minutos al día por 7 días (Figura 4C, D). Posterior a la aplicación de blanqueamiento casero, se procede a la toma del color obtenido con colorímetro; (Chromascop ${ }^{\circledR}$,

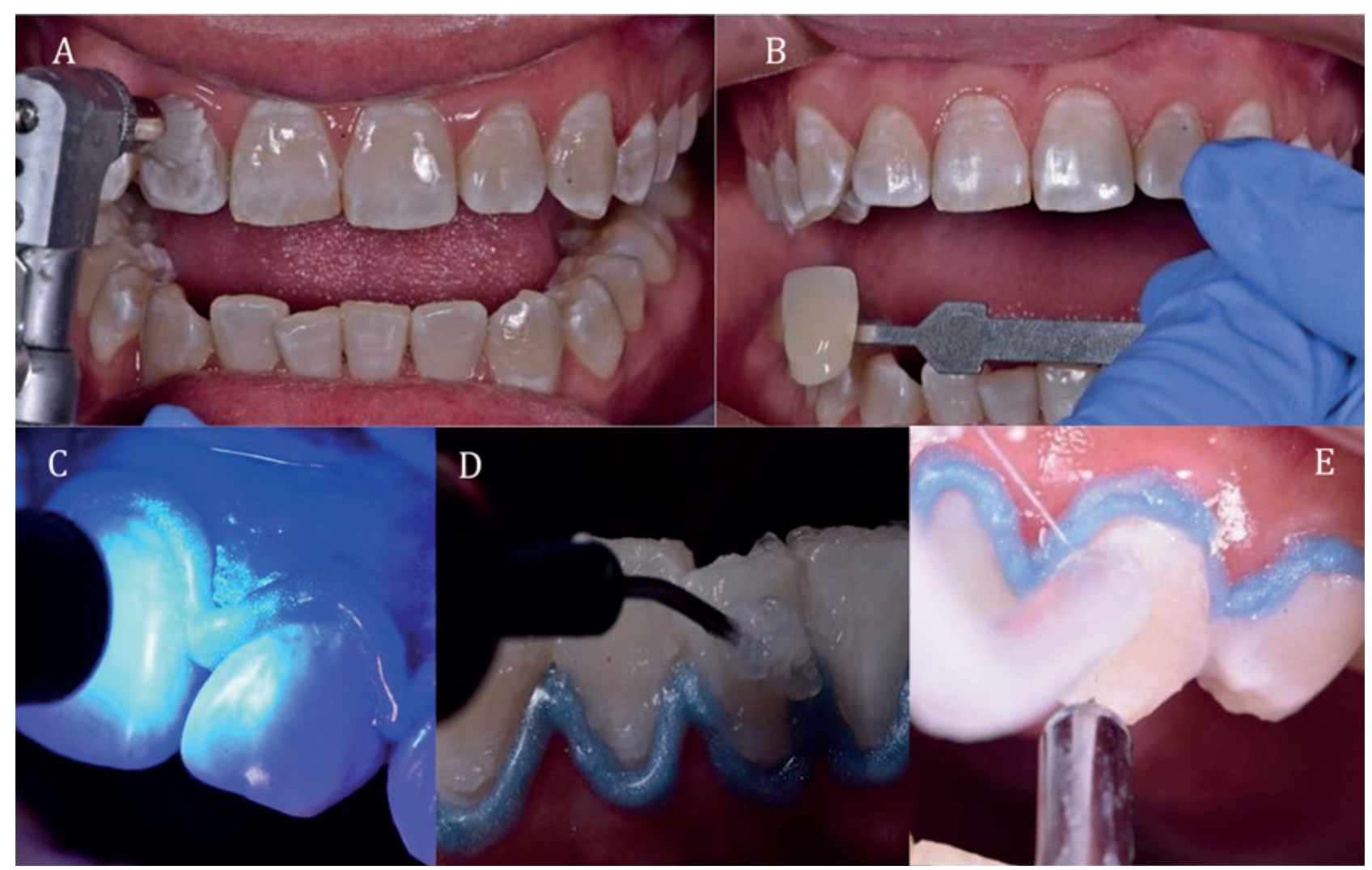

Figura 2. Blanquemiento clínico. A. Profilaxis realizada, previa a la aplicación del blanqueamiento. B. Toma de color. C. Colocación de barrera gingival. D. Aplicación del blanqueamiento clínico, peróxido de hidrógeno al $40 \%$ (ultradent, USA). E. Retiro del agente blanqueador 


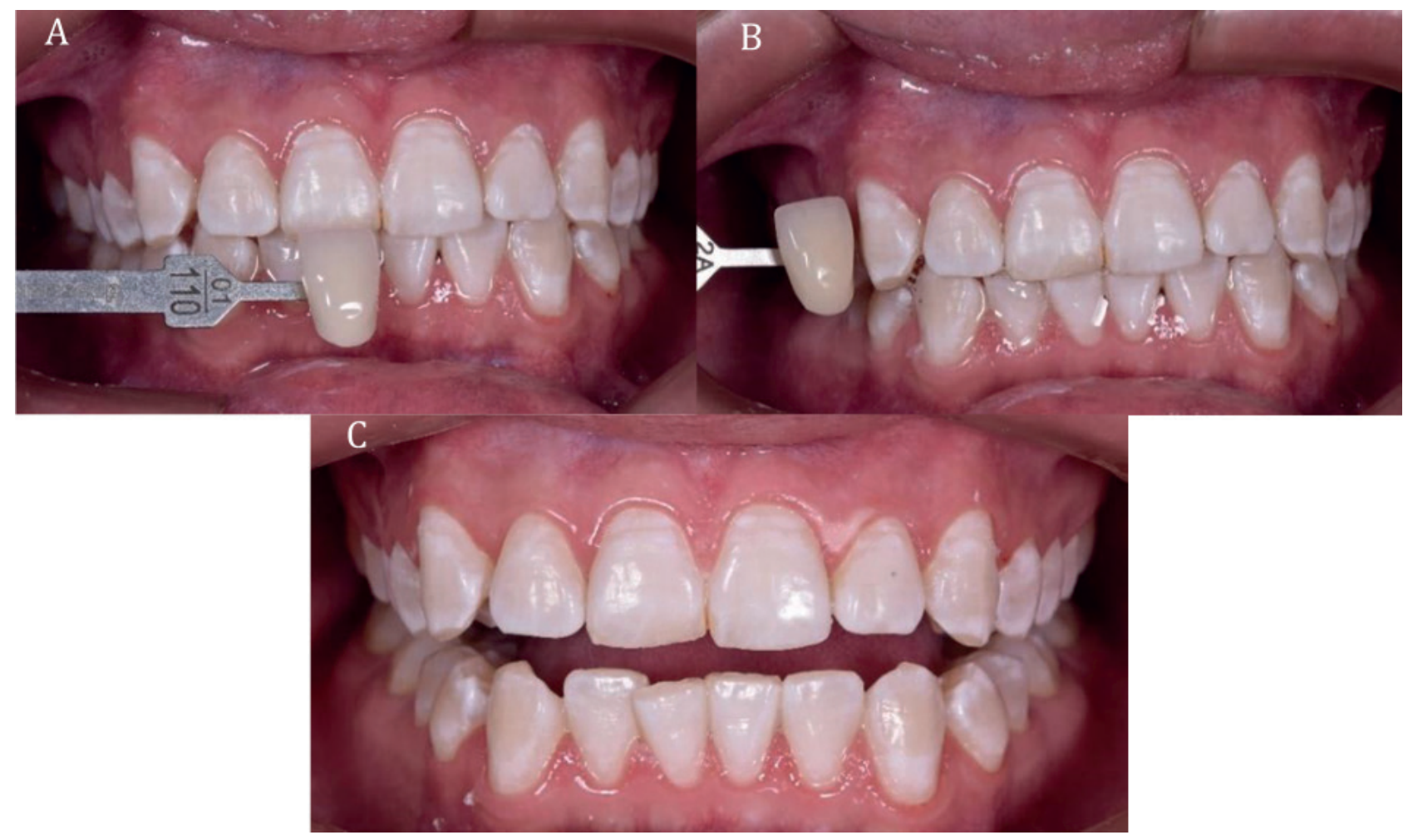

Figura 3. Toma de color después de la aplicación del blanqueamiento clínico. A. Registro de color obtenido después del blanqueamiento clínico 01. B. Registro de color con el color obtenido antes del blanqueamiento clínico 2A. C. Resultado postblanqueamiento clínico

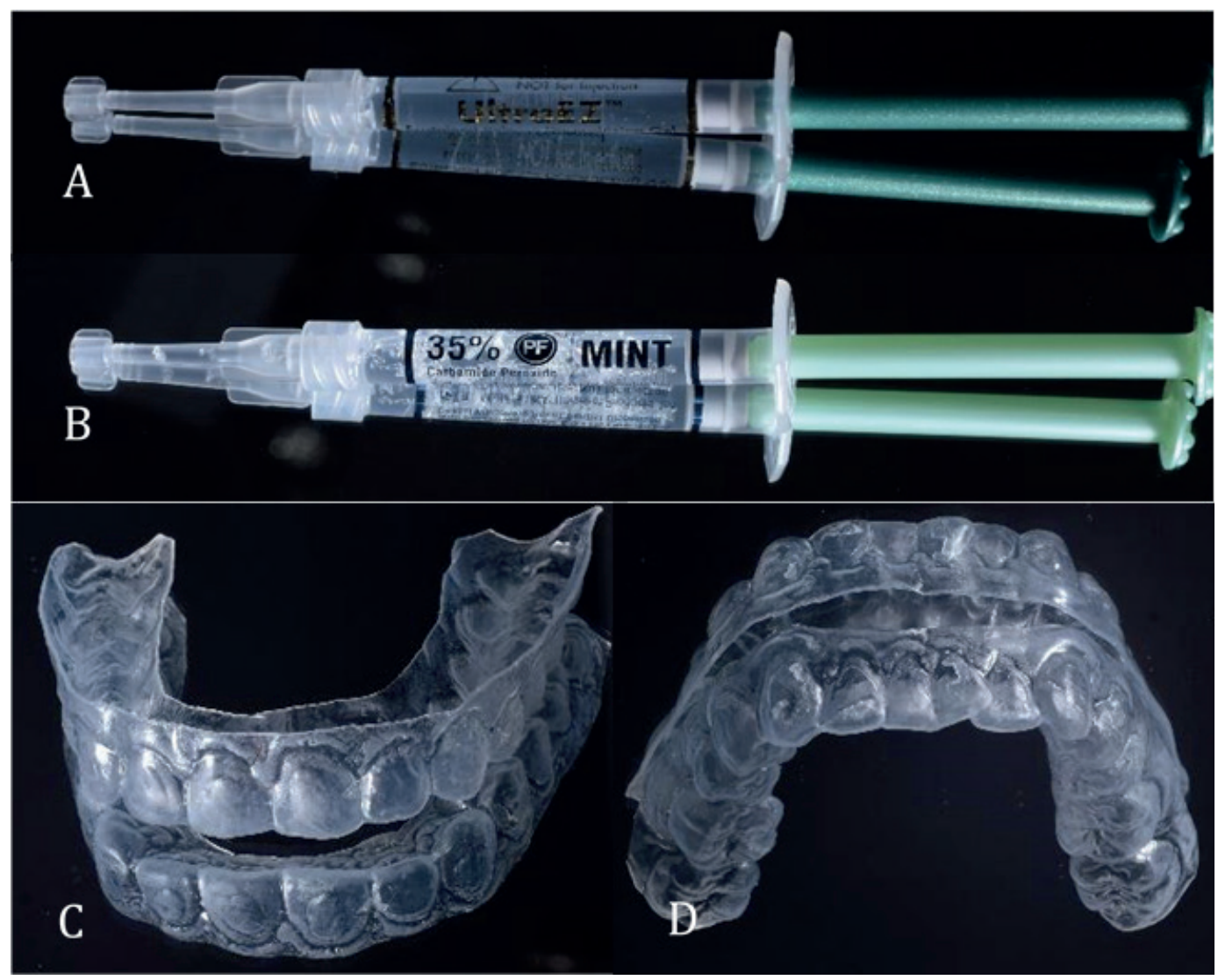

Figura 4. Blanqueamiento casero. A. Desensibilizante ultraez (ultradent, USA). B. Peróxido de carbamida al 35\% (ultradent, USA). C. Acetato superior para aclaramiento casero. D. Acetato inferior para aclaramiento casero 
Ivoclar Vivadent, USA), (Figura 5A, B, C), en esta cita se aplicó por segunda ocasión flúor barniz (Clinpro®, 3M ESPE), buscando una remineralización dental. Se pudo apreciar que el blanqueamiento casero ha dado resultados satisfactorios tanto para el clínico como para el paciente, por lo cual se da por terminada la fase de blanqueamiento (Figura 5). Concluido este proceso, el paciente recibió las recomendaciones respecto al tipo de dieta e higiene para el mantenimiento del tratamiento realizado. A los 27 días de haber finalizado el tratamiento aclarador, fue realizado el control post blanqueamiento, registrando nuevamente el color obtenido (Figura 6). Se programó tres citas más cada 3 meses, para la aplicación de flúor barniz (Clinpro®, $3 \mathrm{M}$ ESPE), con el objetivo remineralizar y tratar las hipomineralizaciones causadas por la FD.

\section{Discusión}

Dentro de los componentes estéticos de una persona, la sonrisa constituye uno de los elementos fundamentales para su comunicación y relacionamiento con su entorno social ${ }^{26}$, ésta puede ser capaz de transmitir el estado de
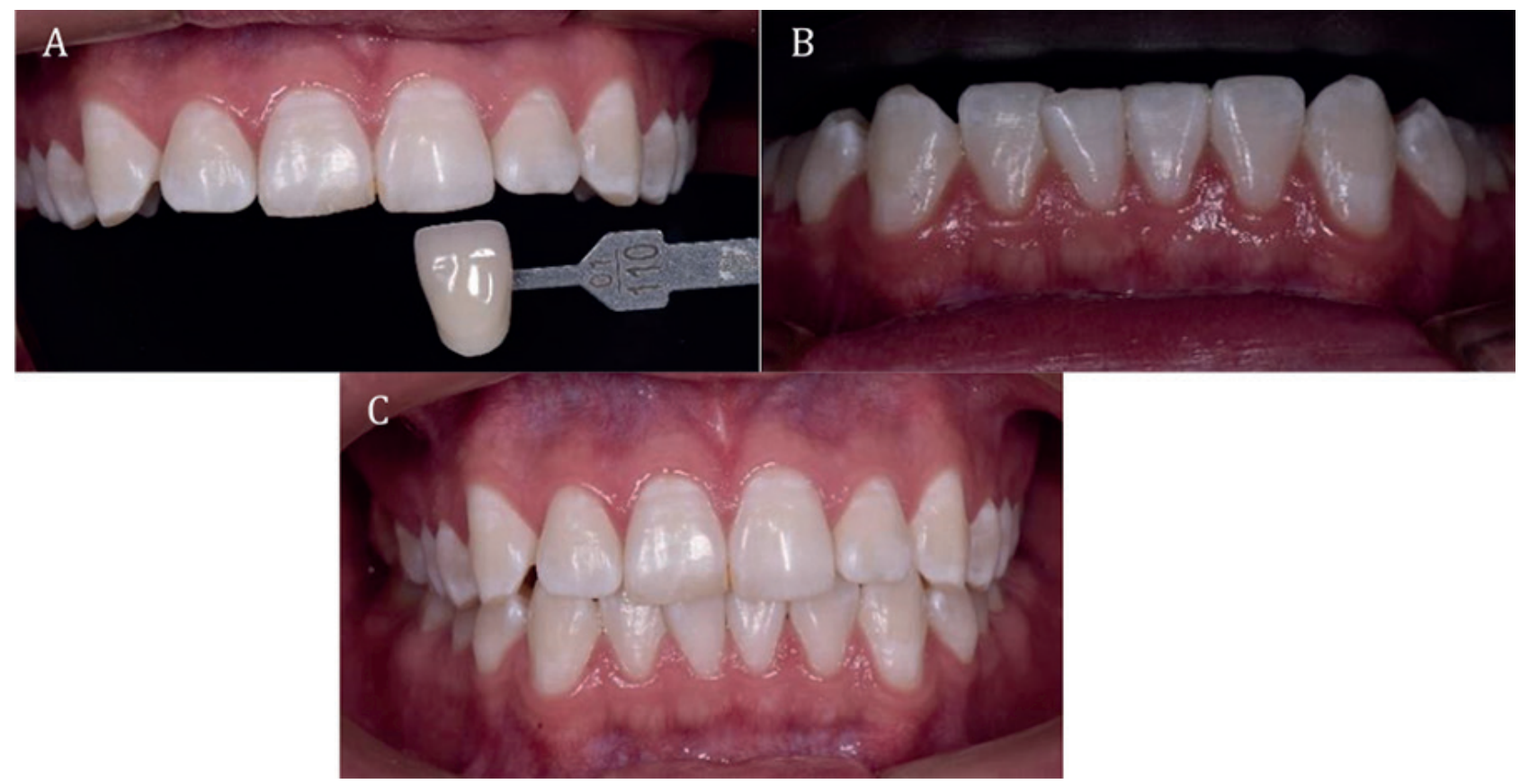

Figura 5. Control a los 8 días de aplicación de blanqueamiento ambulatorio. A. Registro de color arcada superior. B. Registro de color arcada inferior. C. Registro de color obtenido

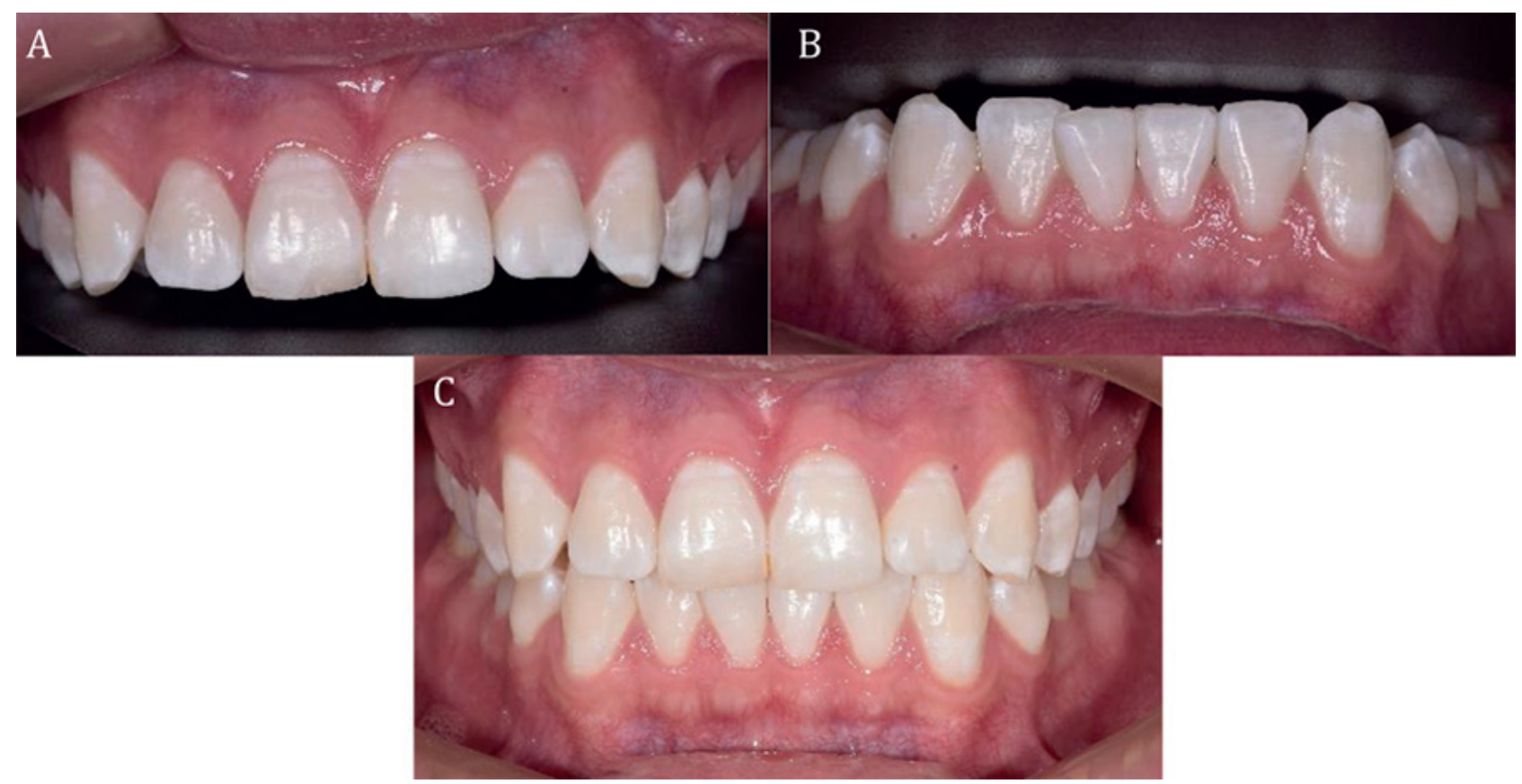

Figura 6. Control a los 27 días concluido el blanqueamiento. A. Registro de color arcada superior. B. Registro de color arcada inferior. C. Registro de color final obtenido 
ánimo de un individuo ${ }^{27}$. La FD generalmente es asintomática, pero desarrolla impacto psicológico negativo dentro de las personas, especialmente en jóvenes ${ }^{28-30}$.

La FD, generalmente se informa como un trastorno del desarrollo del esmalte, es producida por la ingesta excesiva de fluoruros en periodos críticos del desarrollo dental $^{31}$, la exposición a fluoruros entre los 15-30 meses de edad, se considera un periodo crítico para el desarrollo de fluorosis en los dientes permanentes con mayor afectación estética. El desarrollo de la FD, está en función de varios factores asociados a la cantidad de fluoruro, del estado de desarrollo dentario durante el tiempo de exposición y de la variación en la susceptibilidad individual; por lo cual es importante conocer al paciente para saber si ha sido expuesto a fluoruros y con qué frecuencia ${ }^{32}$. En el caso el paciente nos relata que residente desde su nacimiento en una provincia considerada endémica de fluorosis. Actualmente, la presencia de FD en ciertas regiones del Ecuador es muy evidente, la cual puede deberse a varios factores, entre ellos el agua pública de consumo, e incluso el consumo accidental de pasta dental durante el cepillado dental, pudiendo concluir que esta prevalencia irá aumentando, de no ejecutarse medidas de salud pública apropiadas ${ }^{33}$.

El diagnóstico de FD puede resultar muy difícil, los procedimientos recomendados para realizar el diagnóstico diferencial básicamente consisten en distinguir entre opacidades simétricas y asimétricas, análisis fotográfico y la transiluminación. La FD es frecuentemente confundida con otros defectos en el desarrollo del esmalte como la amelogénesis imperfecta, hipoplasia del esmalte, hipocalcificaciones, decoloraciones esmalte causado por tetraciclinas u otros agentes químicos ${ }^{34}$.

Existen diversos grados de FD, actualmente el índice más aceptado a nivel mundial para su clasificación es el índice TF, el cual resulta importante a la hora de seleccionar el tratamiento más indicado para la resolución del caso según su severidad. Diferentes alternativas clínicas han sido desarrolladas para corregir las alteraciones desencadenantes de la FD, todos ellos en dependencia del grado de compromiso dental ${ }^{35}$. En los casos de TF1 al TF3 generalmente será suficiente realizar un blanqueamiento, a menudo asociado a microabrasión ${ }^{36}$. El blanqueamiento dental constituye una de las alternativas más adecuadas por ser un tratamiento conservador y con resultados a corto plazo, es una buena opción para mejorar las propiedades en cuanto al color del esmalte fluorado ${ }^{9}$, proporciona resultados altamente satisfactorios. Algunos estudios han demostrado que el blanqueamiento solo es suficiente para mejorar los resultados estéticos ${ }^{19,37}$.

La eficacia del blanqueamiento dental depende del tiempo de contacto con el diente y de la concentración del ingrediente activo en el producto blanqueador. A mayor concentración de producto blanqueador menor tiempo de exposición ${ }^{38}$. Los agentes blanqueadores usados son el peróxido de hidrógeno, en concentraciones que van desde el 3 al 50\%, y el peróxido de carbamida en concentraciones comprendidas desde el 1 al 45\% ${ }^{39}$.
Un estudio evidenció que la técnica de blanqueamiento dental en consultorio con peróxido de Hidrógeno al 40\% (Opalescence Boost) dio resultados favorables, ya que su efecto aclarador fue notable en todos los casos, con un rango menor de sensibilidad tipo 1 en el $14 \%$ de los casos ${ }^{40}$.

El blanqueamiento clínico por lo general se realiza con concentraciones altas de peróxido de hidrógeno o peróxido de carbamida, en el presente caso, el blanqueamiento clínico se realizó con peróxido de hidrógeno al $40 \%$ de pH neutro, con activación química única, con fluoruro de sodio y nitrato de potasio, que ha demostrado reducir la susceptibilidad a caries, disminuir la sensibilidad y mejorar la microdureza del esmalte. Algunas revisiones sistémicas y meta-análisis mencionan que la intensidad en la sensibilidad dental se asocia cuando el procedimiento se realiza con activación de luz ya que ésta puede aumentar la cantidad de radicales libres que llegan a la pulpa, lo que conduce a una respuesta inflamatoria más intensa y desencadenante del dolor ${ }^{41}$. En este caso no se utilizó activación de luz por dicho sustento y porque el material aplicado no recomendaba su utilización.

La sensibilidad dentaria es uno de los temas con mayor énfasis al someterse a un procedimiento de blanqueamiento dental y al cual los pacientes más relacionan con este procedimiento. Varios estudios han demostrado que la sensibilidad se presenta alrededor del 70\% de los pacientes siendo esta con síntomas leves que permanecen por menos de 24 horas y dura entre 1 a 5 días, el otro $30 \%$ de los pacientes no presentan sensibilidad ${ }^{42}$. En el caso presentado, se evidenció sensibilidad dental durante las primeras 32 horas, la cual se trató con un desensibilizante a base de nitrato de potasio, mostrando resultados efectivos.

Varios estudios demuestran, que el uso de blanqueamiento casero con el uso de concentraciones bajas de peróxido de carbamida con una aplicación diaria de 2 a 3 horas, consigue un blanqueamiento satisfactorio entre 2 - 4 semanas $^{39}$. En este caso posterior a las 48 horas del blanqueamiento clínico, se envió al paciente gel aclarador a base de peróxido de carbamida al 35\%, pero con disminución de tiempo en su aplicación diaria, de 20 minutos por 1 semana, realizando una variación la cual dio resultados efectivos. El flúor dental en presentación de barniz es recomendado como un agente efectivo para el tratamiento de hipersensibilidad dentinaria, produce una barrera física al cubrir y ocluir los túbulos dentinarios, crea una barrera química al formar minerales insolubles dentro de los túbulos dentinarios y gracias a su fórmula de liberación de flúor, calcio y fosfato promueve la remineralización dental ${ }^{43}$. Por lo cual en el paciente se aplicó flúor barniz post blanqueamiento logrando una reducción inmediata de la sensibilidad dental y tratando al mismo tiempo la zona hipomineralizada causada por la FD, sin embargo, se programaron más citas para continuar con la aplicación y dar efectividad al tratamiento e ir controlando al paciente. 
La aplicación de blanqueamiento clínico y domiciliario suele combinarse para lograr mejores resultados en el cambio de tonalidad de los dientes y así garantizar estética al paciente disminuyendo efectos adversos como la sensibilidad dental. La estética y satisfacción en el paciente fue posible alcanzar con la ejecución de procedimientos mínimamente invasivos, respetando la integridad dental, mediante procedimientos de blanqueamiento clínico y casero, siendo un procedimiento actual, innovador y respaldado por literatura científica ${ }^{44}$, destacando que todo tratamiento estará indicado, en dependencia del grado de afectación dental, dejando claro al paciente su compromiso y actuación en el proceso; en el presente caso no se realizaron procedimientos de microabrasión frecuentemente indicados en el tratamiento de la fluorosis leve y moderada ${ }^{38}$, logrando un tratamiento estético y mejorando la calidad de vida en el paciente que padece esta patología, siendo el procedimiento ejecutado un método mínimamente invasivo, con resultados satisfactorios a largo plazo.

La concientización de las necesidades estéticas por parte del paciente previo a cualquier procedimiento odontológico resulta vital para encontrar un punto de equilibrio y satisfacción en el resultado final. La combinación del blanqueamiento de peróxido de hidrógeno al $40 \%$ aplicado en consultorio y peróxido de carbamida al 35\% usado en casa con controles periódicos y aplicación de agentes fluorados por parte del clínico, es el tratamiento idóneo para pacientes con fluorosis grado 3 según el índice de TF. Como plan de tratamiento alternativo se puede efectuar una microabrasión acompañada de blanqueamiento domiciliario, siendo un tratamiento mínimamente invasivo, que requiere ser controlado por el clínico durante su aplicación y además no resulta costoso para el paciente.

\section{Referencias bibliográficas}

1. DenBesten P, Li W. Chronic fluoride toxicity: Dental fluorosis. Monogr Oral Sci [Internet]. 2011 [citado 2020 Abr 8]; 22(1): 81-96. Disponible en: https://www.ncbi. nlm.nih.gov/pubmed/21701193

2. Cavalheiro J, Girotto B, Restrepo M, Bullio C, Loiola R, Escobar A, et al. Aspectos clínicos de la fluorosis dental de acuerdo con las características histológicas: una revisión del Índice de Thylstrup Fejerskov. CES Odontol [Internet]. 2017 [citado $2020 \mathrm{Abr}$ 8]; 30(1):41-50. Disponbile en: http://revistas.ces.edu.co/index.php/odontologia/article/view/4393

3. Hidalgo G, Duque R, Mayor H, Zamora D. Fluorosis dental: no solo un problema estético. Rev Cubana Estomatol [Internet]. 2007 Dic [citado 2020 Mayo 14]; 44(4). Disponible en: http://scielo.sld.cu/scielo.php?script=sci_ arttext\&pid=S0034-75072007000400014\&lng=es.

4. Díaz S, Tirado L, Tamayo G. Impacto de la sonrisa sobre calidad de vida relacionada con salud bucal en adultos. Rev Clin Perio Implantol Rehabil Oral [Internet]. 2018 [citado 2020 Abr 10]; 11(2):78-83. Disponible en: https://www.researchgate.net/publication/327148071_ Impacto_de_la_sonrisa_sobre_calidad_de_vida_relacionada_con_salud_bucal_en_adultos
5. González B, Solórzano P, Balda Z. Estética en odontología: Parte I Aspectos psicológicos relacionados a la estética bucal. Acta Odontol Venez [Internet]. 1999 Dic [citado 2020 Abr 06]; 37(3):33-38. Disponible en: http://www.scielo.org.ve/scielo.php?script=sci_arttext\&pid=S0001-63651999000300008\&lng=es.

6. Thylstrup A, Fejerskov O, Mosha HJ. A polarized light and microradiographic study of enamel in human primary teeth from a high fluoride area. Arch Oral Biol [Internet]. 1978 [Citado 2020 Ene 08]; 23(5):373-80. Disponible en: http://www.ncbi.nlm.nih.gov/pubmed/278575

7. Pereira AC, Moreira BH. Analysis of three dental fluorosis indexes used in epidemiologic trials. Braz Dent J [Internet]. 1999 [Citado 2019 Ene 18];10(1):29-37. Disponible en: http://www.ncbi.nlm.nih.gov/pub$\mathrm{med} / 1086338$

8. Rozier RG. Epidemiologic Indices for Measuring the Clinical Manifestations of Dental Fluorosis: Overview and Critique. Adv Dent Res [Internet]. 1994 [Citado 2020 Abr 24];8(1):39-55. Disponible en: http://journals.sagepub.com/doi/10.1177/089593749400800109 01

9. Di Giovanni T, Eliades T, Papageorgiou SN. Interventions for dental fluorosis: A systematic review. J Esthet Restor Dent [Internet]. 2018 [Citado 2020 Abr 24]; 30(6):502-508. Disponible en: https://scihub.tw/https://www.ncbi.nlm.nih.gov/pubmed/30194793

10. Allibone R, Cronin SJ, Charley DT, Neall VE, Stewart RB, Oppenheimer C. Dental fluorosis linked to degassing of Ambrym volcano, Vanuatu: a novel exposure pathway. Environ Geochem Health [Internet]. 2012 [Citado 2020 Abr 24]; 34(2): 155-170. Disponible en: https://sci-hub.tw/10.1016/j.jdent.2019.103228

11. Abanto JA, Rezende KMPC, Marocho SMS, Alves FBT, Celiberti P, Ciamponi AL. Dental fluorosis: Exposure, prevention and management. Med Oral Patol Oral Cir Bucal. [Internet]. 2009 [Citado 2020 Abr 24]; 14 (2):E103-7. Disponible en: http://www.medicinaoral. com/pubmed/medoralv14_i2_p103.pdf

12. Castro KS, de Araújo Ferreira AC, Duarte RM, Sampaio FC, Meireles SS. Acceptability, efficacy and safety of two treatment protocols for dental fluorosis: A randomized clinical trial. J Dent [Internet]. $2014 \mathrm{Ag}$ [citado 2020 Abr 18]; 42(8): 938-944. Disponible en: https://www. ncbi.nlm.nih.gov/pubmed/24486675

13. Garcia GE. Dental fluorosis: conservative alternative for its treatment. Clinical case. Uncuyo. [Internet]. 2016. [Citado 2020 Abr 24]; 10(1): 28-33. Disponible en: https://bdigital.uncu.edu.ar/objetos_digitales/10729/garciacrimirfo-1012016.pdf

14. Kutuk ZB, Ergin E, Cakir FY, Gurgan S. Effects of in-office bleaching agent combined with different desensitizing agents on enamel. J Appl Oral Sci [Internet]. 2018 [Citado 2020 Ene 08]; 27:e20180233. Disponible en: http://www.ncbi.nlm.nih.gov/pubmed/30427477

15. Gallego G, Zuluaga O. Combinación de tres técnicas de blanqueamiento en dientes no vitales. Reporte de un caso [Internet]. 2006 [Citado 2020 Ene 08]. Disponible en: http://revistas.ces.edu.co/index.php/odontologia/article/viewFile/163/146 
16. Uzcátegui J, Macuar RJP. Alternativa conservadora para el tratamiento de la fluorosis dental de severidad moderada: presentación de un caso. Rev Odontol de los Andes. [Internet] 2014 [Citado 2020 Feb 03]; (7):54-61. Disponible en: http://erevistas.saber.ula.ve/index.php/ odontoula/article/view/7095

17. Castro KS, de Araújo Ferreira AC, Duarte RM, Sampaio FC, Meireles SS. Acceptability, efficacy and safety of two treatment protocols for dental fluorosis: A randomized clinical trial. J Dent [Internet]. 2014 [Citado 2020 Ene 02]; 42(8):938-44. Disponible en: http://www.ncbi. nlm.nih.gov/pubmed/24486675

18. Ramos LR, Fentanes EP, Montiel RN, Dib Kanán A, Esquivel BEE. Tipos y técnicas de blanqueamiento dental. Oral [Internet]. 2007 [Citado 2019 Ene 26]; 8(25):3925. Disponible en: http://www.medigraphic.com/cgi-bin/ new/resumenI.cgi?IDARTICULO $=25998$

19. Briceńo Y, González J, Lara R, Molina M, Paredes O. Efectividad de los Blanqueamientos Dentales: artículo de revisión. Rev Venez Invest Odont [Internet]. 2013 [citado 2020 Ene 12]; 1(2):136-152. Disponible en: http://erevistas.saber.ula.ve/index.php/rvio/article/ view/4592/4367

20. Luque M, Reis A, Schroeder M, et al. Comparison of efficacy of tray-delivered carbamide and hydrogen peroxide for at-home bleaching: a systematic review and meta-analysis. Clin Oral Invest [Internet]. 2016 [citado 2020 May 15]; 20(7):1419-33. Disponible en: https:// doi.org/10.1007/s00784-016-1863-7

21. Kashima T, Tsujimoto Y, Kawamoto K, Senda N, Ito K, Yamazaki M. Generation of Free Radicals and/or Active Oxygen by Light or Laser Irradiation of Hydrogen Peroxide or Sodium Hypochlorite. J Endod. [Internet]. 2013 [citado 2020 Ene 12]; 29(2):141-3. Disponible en: https://pubmed.ncbi.nlm.nih.gov/12597716/

22. Achachao K, Yileng L. Terapias para disminuir la sensibilidad por blanqueamiento dental. Rev. Estomatol. Herediana. [Internet] 2019 [Citado 2020 Abr 24]; 29(4). Disponible en: http://www.scielo.org.pe/scielo.php?pi$\mathrm{d}=$ S1019-43552019000400007\&script=sci_arttext

23. Acuña ED, Vilchez K, Delgado L, Tay LY. Resolviendo mitos sobre indicaciones al paciente durante el blanqueamiento dental. Rev Estomatol Herediana. [Internet] 2015 [Citado 2020 Abr 24]; 25(3):232-7. Disponible en: https://revistas.upch.edu.pe/index.php/REH/article/ view/2616/2515

24. Cano S, Zea M. Manejar las emociones, factor importante en el mejoramiento de la calidad de vida. Rev Logos C \& Tec [Internet]. 2012 [Citado 2020 Abr 10]; 4(1): 58-67. Disponible en: http://www.redalyc.org/ pdf/5177/517751763003.pdf

25. Salazar M, Jácome C. Prevalencia de fluorosis dental ydeterminación del grado de severidad enniños de 6 a 15 años en la Florícola Valleflorubicada en el Valle de Tumbaco. Od.Inv. [Internet]. 2015 [citado 2020 May 15]. 1(1):22-27. Disponible en: https://revistas.usfq.edu.ec/ index.php/odontoinvestigacion/article/view/93/95

26. Barragán E, Ahmad R, Morales M, Itzel C. Psicología de las emociones positivas: generalidades y beneficios. Rev CNEIP [Internet]. 2014 [citado 2020 Abr 10]; 19(1):
103-118. Disponible en: http://www.redalyc.org/articulo.oa?id=29232614006

27. Meireles SS, Goettems ML, Castro KS, Sampaio FC, \& Demarco FF. Dental Fluorosis Treatment Can Improve the Individuals' OHRQoL? Results from a Randomized Clinical Trial. Braz Dent J [Internet]. 2018 [citado 2020 Abr 10]; 29(2), 109-116. Disponible en: https://sci-hub. tw/https://www.ncbi.nlm.nih.gov/pubmed/29898055

28. Browne D, Whelton H, O'Mullane D, Tavener J, \& Flannery E. The aesthetic impact of enamel fluorosis on Irish adolescents. Community Dent Oral Epidemiol. [Internet]. 2011 [citado 2020 Abr 10]; 39(2):127-136. Disponible en: https://sci-hub.tw/10.1111/j.16000528.2010.00577.x

29. Pan ZK, Liu J, Sun G, Chen Y, Wang L, Liu Y, et al. Effects of at-home bleaching and resin infiltration treatments on the aesthetic and psychological status of patients with dental fluorosis: A prospective study. J Dent. [Internet]. 2019 [Citado 2020 Abr 10]. Disponible en: https://sci-hub.tw/10.1016/j.jdent.2019.103228

30. Olivares D, Arellano MJ, Cortés J, Cantín M. Prevalencia y Severidad de Fluorosis Dental y su Asociación con Historia de Caries en Escolares que Consumen Agua Potable Fluorurada en Temuco, Chile. Int J Odontostomatol [Internet]. 2013 [citado 2020 Abr 10]; 7(3):447-454. Disponible en: https://scielo.conicyt.cl/scielo.php?scrip$\mathrm{t}=$ sci_arttext\&pid=S0718-381X2013000300018\&ln$\mathrm{g}=\mathrm{en} \& \mathrm{nrm}=\mathrm{iso} \& \mathrm{t} \operatorname{lng}=\mathrm{en}$

31. Levy SM. An update on fluorides and fluorosis. J Can Dent Assoc [Internet]. 2003 [citado 2020 Abr 18]; 69(5): 286-291. Disponible en: https://www.ncbi.nlm. nih.gov/pubmed/12734021

32. Rango T, Vengosh A, Jeuland M, Whitford GM, TekleHaimanot R. Biomarkers of chronic fluoride exposure in groundwater in a highly exposed population. Sci Total Environ [Internet]. 2017 [citado 2020 En 24]; 1(11):596-597. Disponible en: https://www.ncbi.nlm. nih.gov/pmc/articles/PMC5528157/

33. Salazar M, Jácome C. Prevalencia de fluorosis dental y determinación del grado de severidad en niños de 6 a 15 años en la Florícola Valleflor ubicada en el Valle de Tumbaco. Od Invest [Internet]. 2015 [citado 2010 En 24]; 1(1):22-27. Disponible en: http://revistas.usfq.edu. ec/index.php/odontoinvestigacion/article/view/93

34. Curtis A, Levy SM, Cavanaugh J, Warren J, Kolker J, Weber-Gasparoni K. Decline in Dental Fluorosis Severity during Adolescence: A Cohort Study. J Dent Res. [Internet]. 2020 [citado 2020 Abr 25]; 99(4):388-394. Disponibe en: https://www.ncbi.nlm.nih.gov/pub$\mathrm{med} / 32091961$

35. Villarreal P, Villarreal R, Garza H, Elizondo J. Alternativas de tratamiento para la fluorosis dental. Odont Act [Internet]. 2016 [citado 2020 Abr 10]; 13(160): 52-55. Disponibe en: http://www.imbiomed.com.mx/1/1/articulos. php? method=showDetail\&id_articulo $=107962 \& i d$ seccion $=4703 \&$ \&id_ejemplar $=10495 \&$ id_revista $=306$

36. Edith GC. Fluorosis dental: alternativa conservadora para su tratamiento. Caso clínico. [Internet]. 2016 [Citado 2020 Ene 10]; 10(1):28-33. Disponible en: http:// bdigital.uncu.edu.ar/objetos_digitales/10729/garciacrimirfo-1012016.pdf 
37. García G. Fluorosis dental: alternativa conservadora para su tratamiento. Caso clínico. Facultad de odontología. UNCuyo [Internet]. 2016 [citado 2020 Abr 10]. 10(1):28-33. Disponible en: http://bdigital.uncu.edu.ar/ objetos_digitales/10729/garciacrimirfo-1012016.pdf

38. He L, Shao M, Tan K, Xu X, Li J. The effects of light on bleaching and tooth sensitivity during in-office vital bleaching: A systematic review and meta-analysis. J Dent [Internet]. 2012 [Citado 2020 Abr 08]; 40(8):644-653. Disponible en: https://www.ncbi.nlm.nih.gov/pub$\mathrm{med} / 22525016$

39. Berga C, Forner N, Amengual L. Blanqueamiento vital domiciliario: comparación de tratamientos con peróxido de hidrógeno y peróxido de carbamida. Med. oral patol. oral cir.bucal [Internet]. 2006 [citado 2020 Abr 25] ; 11( 1 ): 94-99. Disponible en: http://scielo.isciii.es/scielo.php?script=sci_arttext \&pi$\mathrm{d}=$ S1698-69462006000100021

40. Lee SS, Kwon SR, Ward M, Jenkins W, Souza S, Li Y. A 3 months clinical evaluation comparing two professional bleaching systems of $25 \%$ and $40 \%$ hydrogen peroxide and extended treatment outcome using a power versus a manual toothbrush. J Esthet Restor Dent [Internet]. 2018 [citado 2020 Abr 12]; 31(2):124-131. Disponible en: https://www.ncbi.nlm.nih.gov/pubmed/30478984
41. Medeiros B, Burey A, Matos T, Loguercio A, Reis A. In-office Dental Bleaching With Light vs. Without Light: A Systematic Review and Meta-Analysis. J Dent. [Internet]. 2018 [citado 2020 Abr 12]; 70:1-13. Disponible en: https://pubmed.ncbi.nlm.nih.gov/29289725/?from_ter$\mathrm{m}=$ light+activated+bleaching\&from_filter $=\mathrm{ds} 1 . \mathrm{y} \_5 \&$ from_pos=2

42. Jia Y, Zhang R, Luo HX, Li P, Zhang CL. Study of the effect of fluoride removal agent and microabrasion technology in LED teeth whitening of dental fluorosis. Shanghai Kou Qiang Yi Xue [Internet]. 2016 [citado 2020 Abr 14]; 25(5): 609-612. Disponible en: https:// www.ncbi.nlm.nih.gov/pubmed/28116437

43. Alvarez D, Robles I, Díaz J, Sandoval P. Abordaje terapéutico de la hipomineralización molar - incisal. Revisión Narrativa. Int. J. Odontostomat. [Internet]. 2017 [citado 2020 Mayo 16]; 11(3): 247-251. Disponible en: https://scielo.conicyt.cl/scielo.php?script=sci_arttext\&pid=S0718-381X2017000300247\&lng=es.

44. Uzcátegui J, Pachas R. Alternativa conservadora para el tratamiento de la fluorosis dental de severidad moderada: presentación de un caso. Rev Odontol de los Andes [Internet]. 2012 [citado 2020 Abr 18]; 7(1):54-61. Disponible en: http://erevistas.saber.ula.ve/index.php/ odontoula/article/view/7095 